\title{
The use of a new glycopeptide antibiotic, teicoplanin, in the treatment of bacterial endocarditis
}

\author{
Alison Webster, A.P.R. Wilson, A.H. Williams, T. Treasure ${ }^{1}$ and R.N. Grüneberg \\ Department of Clinical Microbiology, University College Hospital, London WCIE 6AU and 'Department of \\ Cardiothoracic Surgery, Middlesex Hospital, London, UK.
}

\begin{abstract}
Summary: Teicoplanin, a new glycopeptide antibiotic, has been used to treat twelve patients with bacterial endocarditis due to Gram-positive organisms. Teicoplanin has activity against Gram-positive bacteria similar to vancomycin but therapeutic levels are maintained by a single daily dose, given as an intravenous bolus.

Of six patients with native valve infections, two cases, due to viridans streptococci, were successfully treated with teicoplanin alone and two others, caused by Streptococcus faecalis, were cured by combinations including teicoplanin. One of these patients sustained high tone hearing loss during treatment. The remaining two patients were drug addicts with endocarditis due to Staphylococcus aureus which recurred despite repeated multiple therapy. Of six prosthetic valve infections, antibiotic combinations including teicoplanin cured three cases, caused by streptococci. Infection persisted or treatment was curtailed in three cases of Staphylococcus epidermidis endocarditis.

In this small open study, teicoplanin appeared as effective as vancomycin in the treatment of endocarditis but had the considerable advantage of ease of administration.
\end{abstract}

\section{Introduction}

Infective endocarditis is an uncommon disease, but is still associated with considerable morbidity and mortality. More than $80 \%$ of infections are caused by Gram-positive organisms, ${ }^{1}$ for which there are several generally accepted alternative treatments, including beta-lactams, aminoglycosides, rifampicin and vancomycin. The wider use of vancomycin has been limited by fears of nephro-toxicity and oto-toxicity and difficulty of administration.

Teicoplanin is structurally related to vancomycin, and is active against almost all Gram-positive organisms. ${ }^{2}$ In vitro, its activity is similar to vancomycin but its longer serum half-life of 47 hours allows administration as a single daily injection by the intravenous route. ${ }^{2}$ On the strength of these properties, teicoplanin has been used in combination with other antimicrobial agents in the treatment of twelve cases of infective endocarditis.

\section{Methods}

Bacterial isolates from blood cultures were identified in the usual fashion. Minimum inhibitory and bac-

Correspondence: Alison Webster B.Sc., M.B., B.S. Accepted: 2 March 1987 tericidal concentrations (MIC and MBC) of the antibiotics used were determined for all isolates using a two-fold tube dilution method in nutrient broth, supplemented by $10 \%$ horse serum depending upon growth requirements, using an inoculum of approximately $5 \times 10^{5} \mathrm{cfu} / \mathrm{ml}$. Tubes showing no growth were subcultured onto blood agar plates. The MBC was determined as the lowest concentration of the antibiotic killing $99.9 \%$ of the inoculum. Serum bactericidal activity was measured for all patients on each antibiotic combination by a similar technique. Renal and liver function and haematological indices were monitored during treatment, and audiometry was performed during and on cessation of therapy in 8 of the patients. Teicoplanin (Merrell Dow Pharmaceuticals Ltd.) was administered as a loading dose of $400 \mathrm{mg}$, followed by a once daily injection of $200 \mathrm{mg}$ by intramuscular or bolus intravenous injection. Aminoglycoside dosage was adjusted according to serum levels.

\section{Results}

The courses of antibiotic treatment and outcomes in the 12 patients are given in Table I. Five patients (cases $1,9,10,11$ and 12) had received between 10 and 20 
Table I: Treatment regimens and outcome of 12 patients with infective endocarditis

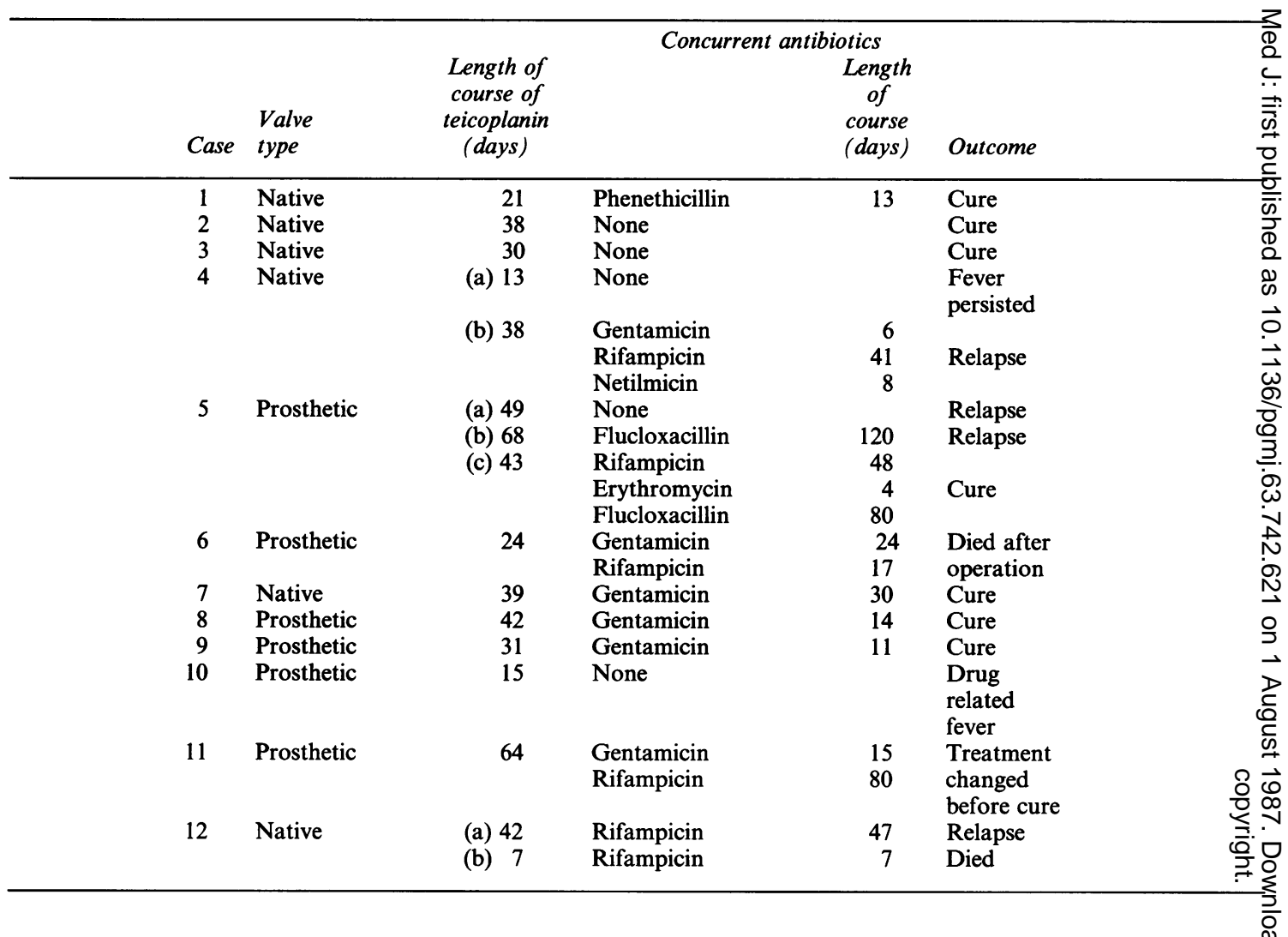

days treatment with other antibiotics, usually a penicillin and aminoglycoside, before changing to teicoplanin. Only one patient (case 1) had become afebrile before starting teicoplanin. Persistence or recurrence of fever, during treatment with teicoplanin, resulted in a change to a different antibiotic combination in two patients (cases 4 and 11) and, in a third (case 5), flucloxacillin was continued for a prolonged period to prevent relapse of prosthetic valve endocarditis.

The organisms isolated and their sensitivities to teicoplanin are given in Table II. Serum bactericidal dilutions were measured in cases $2,3,5$ and 10 in the presence of teicoplanin alone. The staphylococcal isolates were sensitive to methicillin with the exception of the strain of Staphylococcus epidermidis cultured from case 6 .

Adverse reactions were noted in three cases. One patient (case 3), a 39 year old man with Down's syndrome, developed an asymptomatic hearing loss of $30 \mathrm{db}$ at $8 \mathrm{kHz}$ when treated with teicoplanin alone. ${ }^{3}$ Another patient (case 7) showed signs of vestibular and renal damage after treatment but had been exposed to a high level of gentamicin $(>5.1 \mathrm{mg} / \mathrm{l})$ for 3 days at the time of surgical replacement of the valve.
Persistent fever resolved on stopping teicoplaning therapy in one patient (case 10) and reappeared when? it was recommenced. Treatment was successfully흘 completed with clindamycin and fusidic acid.

\section{Discussion}

Despite prolonged parenteral antibiotic therapy, infective endocarditis is associated with a mortality rate of $15 \%{ }^{4}$ and the prognosis for patients witho prosthetic valve infections remains poor. ${ }^{5}$ However, the efficacy of a particular antimicrobial regimen is $\mathrm{S}$ often difficult to assess as other factors, such as $D$ valvular function, may deteriorate despite sterilisation of vegetations. ${ }^{4,6,7}$ Furthermore, comparison of regimens may only be accomplished as part of large multi-centre trials. Nevertheless, an open study, as $\mathrm{O}$ presented here, can indicate the potential usefulness of $\mathrm{W}$ a new antibiotic.

Vancomycin is one of the most commonly usedo antibiotics in the treatment of infective endocarditis due to methicillin-resistant staphylococci or in ${ }^{\infty}$ patients with a history of allergy to penicillin. ${ }^{8}$ Ototox- 
Table II Organisms isolated in the twelve cases of endocarditis. The minimum inhibitory concentration (MIC) and the minimum bactericidal concentration (MBC) of teicoplanin for each organism is given with the maximum bactericidal dilution (MBD) of serum, achieved at trough.

\begin{tabular}{clllll}
\hline Case & Organism & $\begin{array}{c}M I C \\
(m g / l)\end{array}$ & $\begin{array}{c}M B C \\
(\boldsymbol{m g} / l)\end{array}$ & $M B D$ \\
\hline 1 & Strep. faecalis & 0.12 & $>32$ & $1 / 4$ \\
2 & Strep. mitior & 0.12 & - & $1 / 32$ \\
3 & Strep. sanguis & 0.12 & - & $1 / 16$ \\
4 & Staph. aureus & 0.25 & 1.0 & $1 / 8$ \\
5 & Staph. epidermidis & 0.5 & 1.0 & $1 / 32$ \\
6 & Staph. epidermidis & 0.06 & 0.06 & $1 / 32$ \\
7 & Strep. faecalis & 0.12 & 0.25 & $1 / 1$ \\
8 & Strep. group B & 0.06 & 0.06 & $1 / 32$ \\
9 & Strep. bovis & 0.25 & 0.25 & $1 / 64$ \\
10 & Staph. epidermidis & 2.0 & 4.0 & $1 / 2$ \\
11 & Strep. sanguis & 0.12 & 0.25 & $1 / 32$ \\
12 & Staph. aureus & 0.5 & $>8$ & $1 / 2$ \\
\hline
\end{tabular}

icity is rare but renal toxicity is estimated to occur in $5 \%$ of patients when used alone, and in $35 \%$ when used with an aminoglycoside. ${ }^{9}$ Monitoring of serum levels is usually undertaken in an attempt to reduce these risks but minor hearing loss may be overlooked unless serial audiometry is performed. It must also be administered as a slow infusion every $8-12$ hours and phlebitis is troublesome in $13 \%$ of patients. ${ }^{9}$

Teicoplanin, a new glycopeptide antibiotic, has a similar mode of action to vancomycin and is bactericidal at therapeutic concentrations for the majority of Gram-positive bacteria. ${ }^{10,11}$ In a rabbit model of endocarditis, it is bacteriostatic for Streptococcus faecalis when used alone but it shows a marked synergy with gentamicin ${ }^{12}$ and it is as effective as vancomycin against methicillin-resistant Staphylococcus aureus. ${ }^{13}$ However, teicoplanin is potentially a more attractive choice in the treatment of endocarditis because it can be given as a single daily intravenous bolus without causing thrombophlebitis. ${ }^{10}$ Toxicology studies in dogs show that renal damage occurs only at high dosage levels $(40 \mathrm{mg} / \mathrm{kg})$ and is less marked than with vancomycin. ${ }^{10}$

In the present study, all five cases of infective endocarditis caused by penicillin-sensitive streptococci, were cured, including three prosthetic valve infections. Two received teicoplanin as the sole therapy, two received an initial 2 weeks gentamicin therapy, and the fifth patient received prolonged combination chemotherapy. Cure was also achieved in both cases of enterococcal infective endocarditis, although both received gentamicin for 2 to 3 weeks.

The use of teicoplanin appeared less successful in infections caused by staphylococci, despite combination chemotherapy. However, relapses in the two cases of endocarditis due to Staphylococcus aureus were probably related to continued drug abuse and existing therapy for prosthetic valve endocarditis due to Staphylococcus epidermidis also has a poor outcome. ${ }^{5}$ The only patient with infection due to methicillin-resistant staphylococci succumbed despite aggressive chemotherapy.

In this series, the use of teicoplanin resulted in a high tone hearing loss in one patient, which has been reported elsewhere, ${ }^{3}$ and in drug-induced fever in another. However, neither adverse reaction affected the eventual outcome. Vestibular damage in a third patient was more debilitating but, in the presence of high levels of aminoglycoside, the role of teicoplanin was difficult to assess.

Trough bactericidal titres were not helpful in predicting outcome in this study. Weinstein ${ }^{6}$ found that if laboratory variables are controlled, a trough bactericidal titre $>1: 8$ had a predictive value of bacteriological cure of $98 \%$. However, titres of $<1: 8 \mathrm{had}$ a predictive value of only $21 \%$ for failure, and bactericidal titres had no correlation with clinical outcome.

In these 12 cases of infective endocarditis, teicoplanin appears to have been as effective as conventional therapy. It provided a safe and easily administered alternative to vancomycin in the treatment of Gram-positive infection.

\section{Acknowledgements}

We are grateful to Dr R. Davies, Dr R. Emmanuel, Dr A. Hollman, Dr M. Jenkins, Dr P.R. Salmon, Mr M.F. Sturridge, and Dr H. Swanton for permission to summarise the clinical course of patients under their care. Mr D. Felmingham undertook the laboratory control of antibiotic dosage.

A.P.R.W. holds a Research Registrar post generously funded by Merrell Dow Pharmaceuticals, Ltd. 


\section{References}

1. Scheld, W.M. \& Sande, M.A. Endocarditis and intravascular infections. In Mandell, G.L., Douglas, R.G. and Bennett, J.E. (eds) Principles and Practice of Infectious Diseases. John Wiley, New York, 1985, pp 504-530.

2. Verbist, L., Tjandramaga, B., Henrickx, B. et al. In vitro activity and human pharmacokinetics of teicoplanin. Antimicrob Agents Chemother 1984, 26: 881-886.

3. Maher, E.R., Hollman, A. \& Grüneberg, R.N. Teicoplanin-induced ototoxicity in Down's syndrome. Lancet 1986, i: 613 .

4. Malacoff, R.F., Frank, E. \& Andriole, V.T. Streptococcal endocarditis (non enterococcal, non-Group A) single vs. combination therapy JAMA 1979, 241: 1807-1810.

5. Wilson, W.R., Jaumin, P.M., Danielson, G.K., Giuliani, E.R., Washington, J.A. \& Geraci, J.E. Prosthetic valve endocarditis. Ann Intern Med 1975, 82: 751-756.

6. Weinstein, M.P., Stratton, C.W., Ackley, A. et al. Multicentre collaborative evaluation of a standardized serum bactericidal test as a prognostic indicator in infective endocarditis. Am J Med 1985, 78: 262-269.

7. Wilson, W.R., Giuliani, E.R., Danielson, G.K. \& Geraci, J.E. Management of complications of infective endocarditis. Mayo Clin Proc 1982, 57: 162-170.

8. Kucers, A. Vancomycin. J Antimicrob Chemother $1984, \subseteq$ 14: $564-567$.

9. Farber, B.F. \& Moellering, R.C. Retrospective study of $\stackrel{\mathcal{A}}{\stackrel{S}{+}}$ the toxicity of preparations of vancomycin from 1974 to 1981. Antimicrob Agents Chemother 1983, 23: 138-141.

10. Williams, A.H. \& Grüneberg, R.N. Teicoplanin. Antimicrob Chemother 1984, 14: 441-448.

11. Grüneberg, R.N., Ridgway, G.L., Cremer, A.W.L. \& Felmingham, D. The sensitivity of Gram-positive pathogens to teichomycin and vancomycin. Drugs Exptl Clin $\vec{\circ}$ Res 1983, 9: 139-141.

12. Sullam, P.M., Tauber, M.G., Hackbarth, C.J. \& Sande, $\vec{c}$ M.A. Therapeutic efficacy of teicoplanin in enterococcal experimental endocarditis. Antimicrob Agents Chemother 1985, 27: 135-136.

13. Chambers, H.F. \& Sande, M.A. Teicoplanin versus nafcillin and vancomycin in the treatment of experimental endocarditis caused by methicillin-susceptible or -resistant Staphylococcus aureus. Antimicrob Agents $\stackrel{+}{-}$ Chemother 1984, 26: 61-64. 